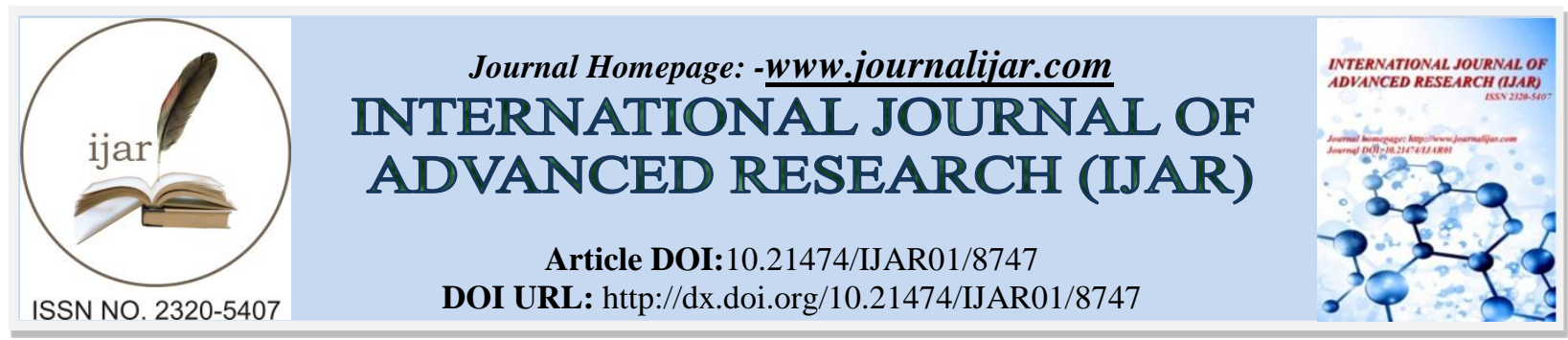

RESEARCH ARTICLE

\title{
MATHEMATICAL MODEL FOR ONE DIMENSIONAL DIFFUSION EQUATION WITHIN THE FIXED LIMITS.
}

\author{
Alpna Mishra. \\ Department of Mathematics, School of Basic Sciences and Research, Sharda University, Greater Noida India- \\ 201306.
}

\section{Manuscript Info}

Manuscript History

Received: 19 January 2019

Final Accepted: 21 February 2019

Published: March 2019

Key words:-

Green's function, Fick's Law, Thermal diffusivity, Thermal conductivity.

\section{Abstract}

A model for the mathematical description of diffusion process is presented through this work and an attempt is also made for the applicability of Green's function method for solving the one dimensional diffusion equation within the desired limits. From this process the required solution to this diffusion equation by considering the initial condition $t=0$ will be obtained. This equation describes the rate of change of concentrations of substances to its own lattice or may be in different substances with a constant diffusion coefficient. At last a computational approach will also be used for getting the numerical solutions. While solving the equation we throughout consider $\mathrm{t}=0$, so that the result may also be applicable in an isothermal diffusion.

Copy Right, IJAR, 2019,. All rights reserved.

\section{Introduction:-}

Diffusion represents the number of atoms that pass a plane from unit area, per unit time in a solute and this flow of solute atoms is called a flux or a current. The flux of solute atoms is driven by some non uniformity or gradient, generally referred to as a force.

The gradient of temperature, momentum and concentrations give rise to corresponding fluxes in thermal energy, momentum and amount of species. We generally consider that diffusion which is generated by a concentration gradient referred to as ordinary diffusion or molecular diffusion. A mathematical model normally may be formulated by using these gradients, specially the concentration gradient [5]. But in this paper more focus is given on the thermal diffusion. The relation between forces and fluxes are the transport constitutive equations. Fourier law for heat transfer, Newton's or Stoke's law for fluid mechanics and Fick's law for mass transfer [2]. The purpose of subject is to solve generic field balance equations energy balance, momentum balance and species balance, with the help of constitutive equations and the particular boundary conditions and initial conditions.

Diffusion may take place within gases, liquids, solids or through their interfaces and always involving a mixture which may be any multi component systems i.e. one with several substances. Diffusion may occur also in two or three dimensions and as far as the two dimensional diffusion is concern it occurs on the surface on solids or along internal surfaces that separate grains of polycrystalline solids known as grain boundary diffusion [1].

There are three levels at which transport phenomena can be studied namely macroscopic level, microscopic level and molecular level [3]. At the macroscopic level we write down a set of equations called the macroscopic balances, 
which describes how the mass, momentum, energy and angular momentum in the system change because of the introduction and removal of these entities via the entering and leaving streams, and because of various other inputs to the systems from the surroundings. No attempt is made to understand all the details of the system. At the microscopic level we examined what is happening to the fluid mixture in a small region within the equipment. We write down a set of equations called the equations of change, which describe how the mass, momentum, energy and angular momentum change within this small region. The aim here is to get the information about velocity, temperature, pressure and concentration profiles within the system. This more detailed information may be required for the understanding of some processes. At the molecular level we seek a fundamental understanding of the mechanism of mass, momentum, energy and angular momentum transport in terms of molecular structure and intermolecular forces. It should be evident that these three levels of descriptions involve different length scales. So that problem of mass transfer for diffusion can also be described by the system of differential equation with boundary conditions and contact conditions.

Mathematical modeling of mass transfer by diffusion in solids and the methods for finding the analytical solutions have been also studied by several authors [6]. At the same time the mathematical modeling of mass transfer in symmetric heterogeneous and non-porous media with a system of n-interface interactions has already discussed [7].

As far as the thermal diffusion is concerned, it simply refers to the transport of atoms driven by the temperature gradient. Unlike ordinary diffusion in concentration gradient, thermal diffusion is not a random process; the affected atoms flow along the temperature gradient, either up or down, thermal diffusion affects impurity atoms in a host crystal.

The mathematical model and formulation:

The diffusion equation is derived by considering concentration gradient. Therefore at any point $\mathrm{x}, \mathrm{y}$, zsome solute is having concentration c per unit volume at time t. Due to the concentration gradient grad c there is a flow of solute atoms from higher concentration to lower concentration which is given by the density vector at that time considered as $\mathrm{j}$. Then according to Fick's first law we have

$\mathrm{J}=-$ Dgradc $=-\mathrm{D} \Delta \mathrm{c}$

The negative sign in the above equation shows that flow is towards the low concentration.

Now, if we consider a surface $\mathrm{S}$ with volume $\mathrm{V}$, then the rate of change of the amount is given by $\frac{\partial}{\partial \mathrm{t}} \int_{\mathrm{V}} \mathrm{c}(\mathrm{x}, \mathrm{y}, \mathrm{z}, \mathrm{t}) \mathrm{dxdydz}$

and the amount of the solute which comes out to the surface $\mathrm{S}$ per unit time is

$\int_{S} \mathrm{j} \cdot \hat{n} \mathrm{ndS}$

where $\mathrm{n}$ is the unit normal vector to the surface.

If there is no sink and source inside the volume, then by equations (2) and (3), we get

$\frac{\partial}{\partial t} \int_{V} c(x, y, z, t) d x d y d z=-\int_{S} \hat{j}$. $\hat{n d} S=\int_{S}($ Dgradc $) \hat{n d} S=\int_{V} \operatorname{div}($ Dgradc $) d x d y d z$

Hence, $\int_{V}\left[\frac{\partial c}{\partial t}-\operatorname{div}(\right.$ Dgradc $\left.)\right] d x d y d z=0$

Since equation (4) hold for all volume, we get the Fick's second law of equation as

$\frac{\partial \mathrm{c}}{\partial \mathrm{t}}=\operatorname{div}($ Dgradc $)$

Further $\mathrm{D}$ is assumed to be a constant and known as diffusion coefficient than we get the diffusion equation

$$
\frac{\partial \mathrm{c}}{\partial \mathrm{t}}=\operatorname{div}(\text { Dgradc })=\mathrm{D} \Delta^{2} \mathrm{c}, \text { where } \Delta^{2} \equiv\left(\frac{\partial}{\partial \mathrm{x}^{2}}+\frac{\partial}{\partial \mathrm{y}^{2}}+\frac{\partial}{\partial \mathrm{z}^{2}}\right)
$$

Hence the equation in one dimensional form becomes, $\frac{\partial c}{\partial t}=\mathrm{D} \frac{\partial^{2} \mathrm{c}}{\partial \mathrm{x}^{2}}$

The equation governing by the temperature gradient heat conduction equation is

$\frac{\partial \mathrm{T}}{\partial \mathrm{t}}=\alpha \Delta^{2} \mathrm{~T}=\alpha \frac{\partial^{2} \mathrm{~T}}{\partial \mathrm{x}^{2}}$

where $\alpha$ is known as thermal diffusivity of the substance.

Analytical description of diffusion process:

Consider the distribution of atoms of substances A to substance B. Diffusion from any point of individual substance can be given by Gaussian function which defines the normal distribution probability function as, $\mathrm{P}(\mathrm{x})=$ $\frac{1}{\sigma \sqrt{2 \pi}} \mathrm{e}^{-\frac{(x-\xi)^{2}}{2 \sigma^{2}}}$

where $\sigma$ is the standard deviation such that $\sigma \in[0,+\infty)$. 
When $\sigma \rightarrow 0$ then $\mathrm{P}(\mathrm{x}) \rightarrow \delta(\xi)$, where $\delta$ defines the Dirac delta function, so that to find the solution of diffusion equation the method of Green's function can be used which is of the following form

$T(x, t)=\int_{-\infty}^{+\infty} G(x, \xi, t) f(\xi) d \xi$,

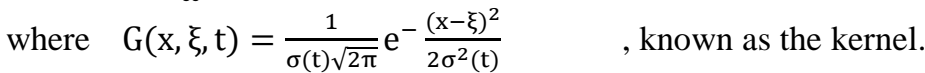

Function $\sigma(\mathrm{t})$ is the diffusion depth or diffusion radius of individual elementary species, for diffusion from one point.

Now from equation (8) and (9) $\quad \mathrm{T}(\mathrm{x}, \mathrm{t})=\int_{-\infty}^{+\infty} \frac{1}{\sigma(\mathrm{t}) \sqrt{2 \pi}} \mathrm{e}^{-\frac{(\mathrm{x}-\xi)^{2}}{2 \sigma^{2}(\mathrm{t})} \mathrm{f}(\xi) \mathrm{d} \xi}$

Hence, $\frac{\partial^{2} \mathrm{~T}}{\partial \mathrm{x}^{2}}=$

$$
\begin{aligned}
\frac{1}{\sigma^{5}(\mathrm{t}) \sqrt{2 \pi}} \int_{-\infty}^{+\infty} \mathrm{e}^{-\frac{(\mathrm{x}-\xi)^{2}}{2 \sigma^{2}(\mathrm{t})}} & {\left[(\mathrm{x}-\xi)^{2}-\sigma^{2}(\mathrm{t})\right] \mathrm{f}(\xi) \mathrm{d} \xi } \\
& \frac{\partial \mathrm{T}}{\partial \mathrm{x}}=\frac{\sigma^{\prime}(\mathrm{t})}{\sigma^{4}(\mathrm{t}) \sqrt{2 \pi}} \int_{-\infty}^{+\infty} \mathrm{e}^{-\frac{(\mathrm{x}-\xi)^{2}}{2 \sigma^{2}(\mathrm{t})}}\left[(\mathrm{x}-\xi)^{2}-\sigma^{2}(\mathrm{t})\right] \mathrm{f}(\xi) \mathrm{d} \xi
\end{aligned}
$$

Also, from equation (10) we have, by taking $\mathrm{t}=0, \mathrm{~T}(\mathrm{x}, 0)=\int_{-\infty}^{+\infty} \delta(\xi) \mathrm{f}(\xi) \mathrm{d} \xi=\mathrm{f}(\xi)$

Since $\int_{-\infty}^{+\infty} \delta(\xi) \mathrm{d} \xi=1$.

$$
\begin{aligned}
& \text { Combining equation } \\
& \sigma^{\prime}(\mathrm{t}) \int_{-\infty}^{+\infty} \mathrm{e}^{-\frac{(\mathrm{x}-\xi)^{2}}{2 \sigma^{2}(\mathrm{t})}}\left[(\mathrm{x}-\xi)^{2}-\sigma^{2}(\mathrm{t})\right] \mathrm{f}(\xi) \mathrm{d} \xi=\frac{\alpha}{\sigma(\mathrm{t})} \int_{-\infty}^{+\infty} \mathrm{e}^{-\frac{(\mathrm{x}-\xi)^{2}}{2 \sigma^{2}(\mathrm{t})}}\left[(\mathrm{x}-\xi)^{2}-\sigma^{2}(\mathrm{t})\right] \mathrm{f}(\xi) \mathrm{d} \xi
\end{aligned}
$$

It gives, $\sigma^{\prime}(\mathrm{t})=\frac{\alpha}{\sigma(\mathrm{t})}$

and $\sigma(\mathrm{t})=\sqrt{2 \alpha \mathrm{t}}$,

Hence, $\quad T(x, t)=\frac{1}{\sqrt{4 \pi \alpha t}} \int_{-\infty}^{+\infty} e^{-\frac{(x-\xi)^{2}}{4 \alpha t}} f(\xi) d \xi$

This is the elementary or fundamental solution of diffusion equation for infinite interval. Also $c(x, t)$ is an analytic function of $\mathrm{x} \& \mathrm{t}$ and is positive for every $\mathrm{x}$.

Let for $\mathrm{t}=0$, initial and final conditions are defined as-

$$
\mathrm{T}(\mathrm{x}, 0)=\left\{\begin{array}{c}
\mathrm{t}_{0}(\text { constant }) \text { fora }<\mathrm{x}<\mathrm{b} \\
\text { Oforoutside }
\end{array}\right.
$$

Taking equation (13) \& (17), we get

$f(\xi)=\left\{\begin{array}{c}t_{0} \text { (constant)for } \xi \leq 0 \\ \text { Ofor } \xi>0\end{array}\right.$

Equation (10) can be rewritten with (15) and (18) as, $\mathrm{T}(\mathrm{x}, \mathrm{t})=\frac{\mathrm{t}_{0}}{\sqrt{4 \pi \alpha \mathrm{t}}} \int_{\mathrm{a}}^{\mathrm{b}} \mathrm{e}^{-\frac{(\mathrm{x}-\xi)^{2}}{4 \alpha \mathrm{t}}} \mathrm{d} \xi$

Introducing the new independent variable $\eta$, defined as- $\eta=-\frac{(x-\xi)}{\sqrt{4 \alpha \mathrm{t}}}$,

hence $\mathrm{d} \xi=\sqrt{4 \alpha \mathrm{t}} \mathrm{d \eta}$

Now equation (19) becomes,

$\mathrm{T}(\mathrm{x}, \mathrm{t})=\frac{\mathrm{t}_{0}}{\sqrt{\pi}} \int_{\frac{(\mathrm{a}-\mathrm{x})}{\sqrt{4 \alpha \mathrm{t}}}}^{\frac{(\mathrm{b}-\mathrm{x})}{\sqrt{\alpha t}}} \mathrm{e}^{-\eta^{2}} \mathrm{~d} \eta=\frac{\mathrm{t}_{0}}{2}\left[\frac{2}{\sqrt{\pi}} \int_{0}^{\frac{(\mathrm{b}-\mathrm{x})}{\sqrt{4 \alpha \mathrm{t}}}} \mathrm{e}^{-\eta^{2}} \mathrm{~d} \eta-\frac{2}{\sqrt{\pi}} \int_{0}^{\frac{(\mathrm{a}-\mathrm{x})}{\sqrt{4 \alpha \mathrm{t}}}} \mathrm{e}^{-\eta^{2}} \mathrm{~d} \eta\right]$

After introducing the error function, which is defined as, $\operatorname{erf}(\mathrm{z})=\frac{2}{\sqrt{\pi}} \int_{0}^{z} \mathrm{e}^{-\eta^{2}} \mathrm{~d} \eta$

the final equation becomes, $T(x, t)=\frac{t_{0}}{2}\left[\operatorname{erf}\left(\frac{b-x}{\sqrt{4 \alpha t}}\right)-\operatorname{erf}\left(\frac{a-x}{\sqrt{4 \alpha t}}\right)\right]$

In the terms of concentration, this result can be restated as,

$C(x, t)=\frac{c_{0}}{2}\left[\operatorname{erf}\left(\frac{b-x}{\sqrt{4 D t}}\right)-\operatorname{erf}\left(\frac{a-x}{\sqrt{4 D t}}\right)\right]$

\section{Numerical results:-}

As we know that the gradient of concentration and temperature defines the corresponding fluxes in amount of species and thermal energy respectively, so that they are referred to as ordinary diffusion and thermal diffusion respectively. The following graphs shows a correspondence between the two results appear in equations (21) and (22), which derives the values of temperature and concentration respectively. Heat conduction in fluids can thought of as molecular energy transport, inasmuch the basic mechanism is the motion of the constituent molecules. It is known that some materials such as metals conduct heat rapidly, whereas other such as wood acts as thermal insulators. The physical property that describes the rate at which heat is conducted is thermal conductivity $\kappa$ and in 
addition the thermal diffusivity a is defined in terms of thermal conductivity $\mathrm{k}$ as $\alpha=\frac{\kappa}{\rho \mathrm{c}_{\mathrm{p}}}$ where $\hat{c_{p}}$ is the heat capacity at constant pressure per unit mass. Also when the assumptions of constant physical properties is made the quantity a i.e. thermal diffusivity occurs with kinematics viscosity $v$ in the similar ways with same dimensions length ${ }^{2} /$ time in the equation of change for momentum and energy transport. It can be also seen that thermal conductivities of gases at low density increases with increasing temperature, but in this work the graphs are drawn by treating density as a constant quantity, whereas the thermal conductivity of most liquids decreases with increasing temperature. In the case when thermal conductivities is considered in solids then it have to be measured experimentally, since they depend on many factors that are difficult to predict or measure. In crystalline materials, the phase and the crystalline size are important, in amorphous solids, the degree of molecular orientation is considerable effect. In porous solids, the thermal conductivity is strongly dependent on the void fraction of the pore size and the fluid contained in the pores. The graphs indicate clearly that when we increase the values of spatial coordinates, the concentration and the temperature increases continuously and it can also be seen that both concentration $\mathrm{C}$ and temperature $\mathrm{T}$ depends highly on initial concentration and initial temperature respectively and both quantities increase with increase in initial values in a constant manner, so that have a same shapes of variations for different parameters appear in the solutions. Figure. 1, 2, 3, 4 are drawn for different diffusion coefficient respectively, which shows that concentration increases as we increase the diffusion coefficient as well as the initial concentration. Same results are coming for the different thermal diffusivities in Figure. 5, 6, 7, 8, with increasing initial temperature.

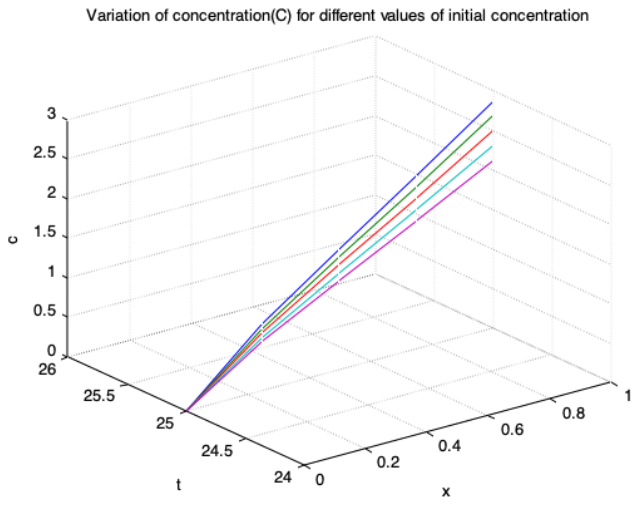

Figure1:-

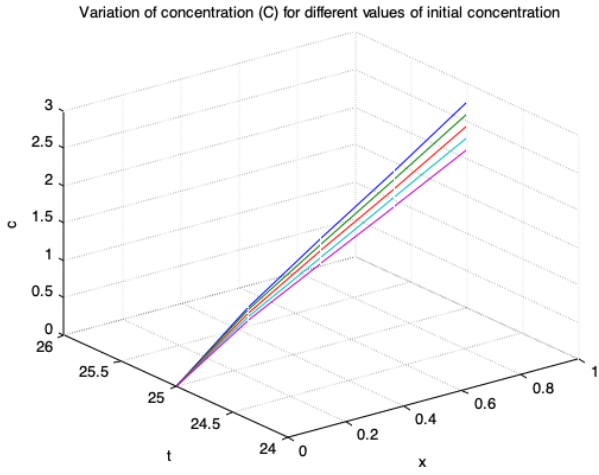

Figure 2:-

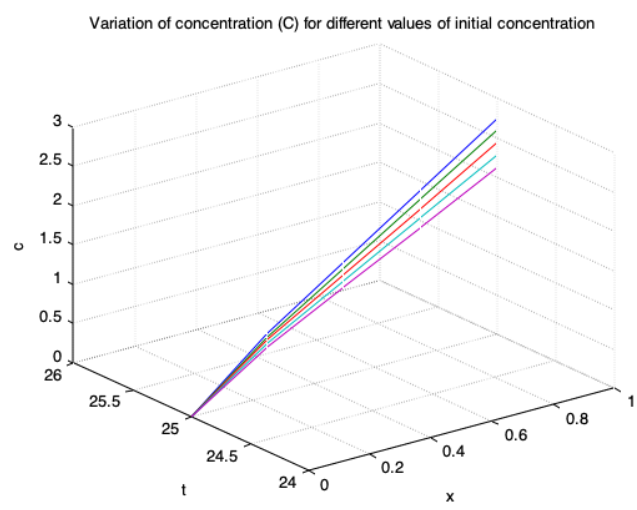

Figure3:-

Variation of concentration(C) for different values of initial concentration

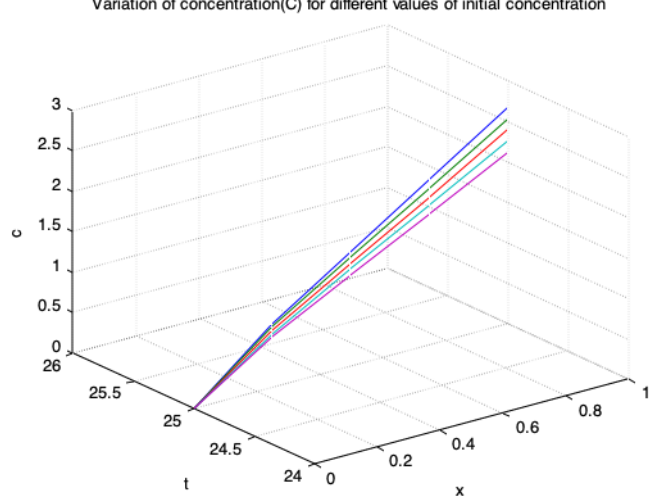

Figure 4:- 


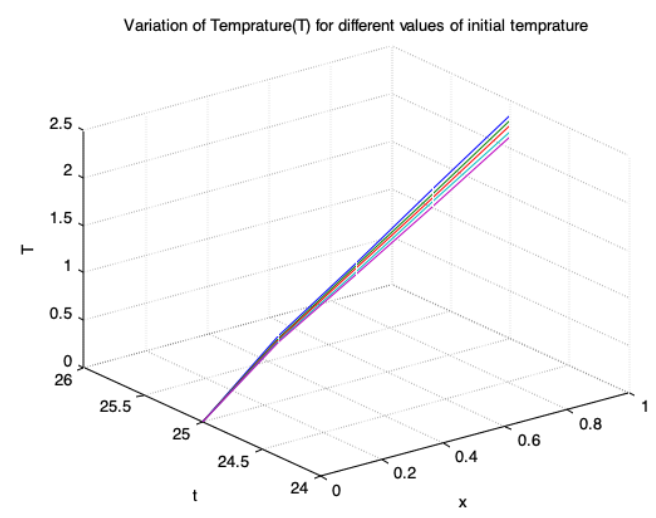

Figure 5:-

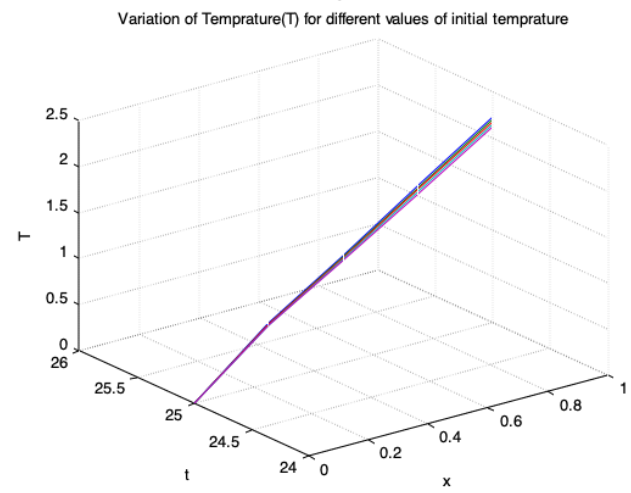

Figure 6:-

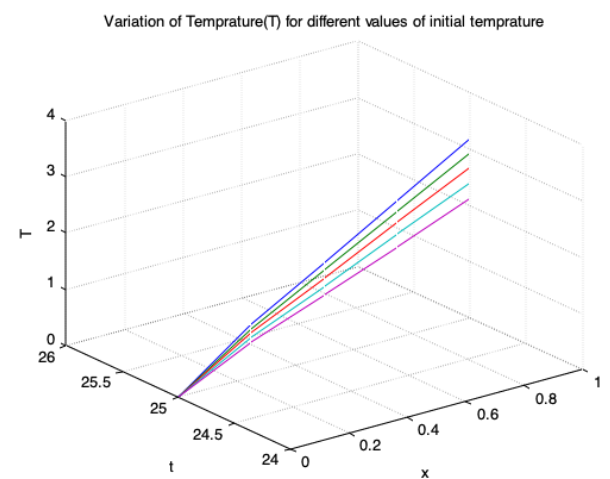

Figure 7:-

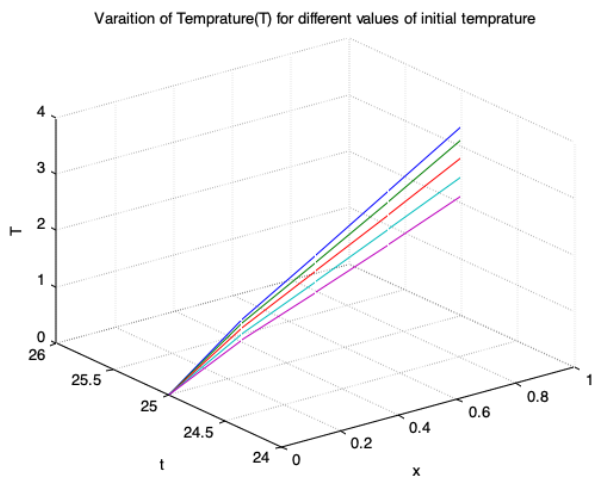

Figure8:-

\section{Conclusion:-}

For the mathematical modeling of diffusion process in substances, we can apply the Fick's second law, which was solved by the applicability of the Green's function. Eq.(21), solved with the initial condition taking $t=0$, is a final result of Eq. (6) and a parallel form in terms of temperature was restated in Eq. (21). The nature of complementary error function used in the final result [Eq. 20] is such that the penetration of solute is effectively limited to depths which is less than or equal to the twice of the diffusion depths. This condition serves to restrict the anneal time in the diffusion experiments when the thickness of the blocks of the solid in some necessary finite value of thickness of solids. In the whole process we take $t=0$, which is a constant so that we can also apply the final result in isothermal diffusion, that is a physical process undergoes in such a way that its temperature remains constant throughout.

\section{Reference:-}

1. Shewmon, P. 1989. Diffusion in solids , $2^{\text {nd }}$ Ed., The Minerals Metals and Materials Society.

2. Bird, R.B., Stewart, W.E. and Lightfoot, E.N. 2002. Transport phenomenon, John Wiley and Sons Asia Pvt. Ltd, India.

3. Boznani, E., Fytianos, K. and Voudrias, E. 2002. Sorption desorption of dyes from aqueous solution and waste waters with different sorbent materials, Global Nest. The International Journal, 41, 178-182.

4. Suchecka, M., Borisovich, A. and Serbimnski, W. 2005. Green's function methods for mathematical modeling of unidirectional diffusion process in isothermal metals bonding process, Advances in Material Science, 5 (38), 7579.

5. Petryk, M., Leclerc, S., Canet, D., and Fraissard, J. 2007. Mathematical modeling and visualization of gas transport in a zeolite bed using a slice selection procedure, Diffusion Fundamentals, 4, 11.1-11.23.

6. Petryk, M.R. 2007. Mathematical modeling of mass transfer in symmetric heterogeneous and non-porous media with a system of n-interface interactions, Cybernetics and Systems Analysis, 43, 94-111.

7. Kumar, S. and Mishra, A. 2009. A mathematical model for the diffusion process in a binary mixture of chemical species, International Journal of Computational Mathematics and Numerical Simulation, 21, 71-78. 
8. Zhang, K. and Wang, S. 2009. A computational scheme for options under jump diffusion processes, International Journal of Numerical Analysis and Modeling, 6 (1), 110-123.

9. Peres,R., Muller,E., Mahajan,V. 2010. Innovation diffusion and new product growth models. A critical review and research directions, International Journal of Research in Marketing, 272, 91-106.

10. Kalis, H., Rogovs, S. and Gedroics, A. 2012. On the mathematical modelling of the diffusion equation with piecewise constant coefficients in a multi layered domain, International Journal of Pure and Applied Mathematics, 84, 555-575.

11. Bonforte, M. Segatti, A. and Vázquez, J.L. 2016. Non-existence and instantaneous extinction of solutions for singular nonlinear fractional diffusion equations, Calc. Var. Partial Differential Equations, 55(3), 1-29.

12.Vazquez, J.L. 2017. The mathematical theories of diffusion. Nonlinear and fractional diffusion, arXiv 1706.08241v1.

13. Kumar S. and Mishra A. 2018. Variation of moisture content in the presence of combined flux, Journal of Advances in Mathematics, 141, 7624-7630.

14.Mishra, A. and Kumar, S. 2018. Diffusion equation model for determining the concentration of urea in artificial kidney, International Research Journal of Advanced Engineering and Science, 3(3), 214-217. 